\title{
Stratification of patients with autoinflammatory phenotypes by interferon (IFN) score suggests a new group of IFN mediated autoinflammatory diseases with overlapping clinical phenotypes
}

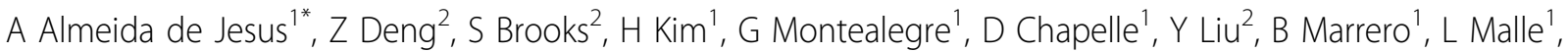 \\ M O'Brien' ${ }^{1}$ W Goodspeed ${ }^{1}$, Y Huang ${ }^{1}$, P Hashkes ${ }^{3}$, G Nasrullayeva ${ }^{4}$, MT Terreri ${ }^{5}$, C Silva ${ }^{6}$, B Arabshahi ${ }^{7}, \mathrm{~K} \mathrm{O}^{\prime}$ Neill $^{8}$, \\ M Punaro ${ }^{9}$, L Moorthy $^{10}$, A Reinhardt $^{11}$, V Lilleby $^{12}$, J Niemela $^{13}$, S Rosenzweig $^{13}$, T Fleisher $^{13}$, \\ R Goldbach-Mansky ${ }^{1,13}$
}

From 8th International Congress of Familial Mediterranean Fever and Systemic Autoinflammatory Diseases Dresden, Germany. 30 September - 3 October 2015

\section{Background}

We have identified mutations in proteasome components as the cause of CANDLE syndrome and in TMEM173/ STING as the cause for a severe vasculopathy and lung disease, SAVI. CANDLE and SAVI patients do not respond to IL-1 inhibition and consistently demonstrate marked up-regulation of IFN-inducible genes. Our data suggest innate immune dysregulation caused by chronic Type I IFN signaling in both conditions.

\section{Objective}

We hypothesize that the presence of IFN signature may identify patients with autoinflammatory disease (AID) who have genetic mutations in other IFN regulating genes.

\section{Methods}

To identify patients with IFN signatures, RNA sequencing (RNA-seq) from whole blood RNA was performed using HiSeq 2000 Illumina ${ }^{\circledR}$ platform. Heatmaps with 64 IFN response genes were assessed. Whole exome sequencing (WES) was performed from whole blood DNA.

\section{Results}

We identified 19 patients with marked upregulation of IFN inducible genes. WES was performed in 14 patients and parents (trios) and in 5 individual patients. Of the probands, 9/19 were female, 8/19 were Caucasian, 3 Asian, 2 Hispanic, 2 Norwegian and 4 had other ethnicities. All patients presented with immunodysregulatory phenotypes with clinical similarities to the previously described interferonopathies, including skin vasculitis/vasculopathy (9/19), panniculitis (12/19), myositis (5/19) and basal ganglion calcifications $(5 / 19)$, but had no genetic diagnosis prior to $\mathrm{NIH}$ evaluation. The bioinformatics variant annotation, analysis and filtering workflow successfully identified mutations in IFN-regulating genes in 7 of the 19 probands. In one patient, we found a disease causing de novo and somatic mutation in TREX1. This patient also presented with an in-frame deletion in $D H X 9$ inherited from her mother and a missense mutation in MAVS inherited from her father. In one patient, we identified a de novo mutation in $D H X 9$ and this patient is also a compound heterozygous for mutations in IFIH1/MDA5. In a third patient, we found a missense mutation in TREX1 inherited from the mother and a heterozygous variant in $M B 21 D 1$ (gene encoding cGAS) inherited from the father. A fourth patient with a clinical phenotype of CANDLE had two novel compound heterozygous mutations in PSMG2. Additionally, a male patient with lupus-like clinical and laboratory findings was found to have an X-linked mutation in TREX2 gene. All mutations described were confirmed by Sanger sequencing.

National Institutes of Health, Translational Autoinflammatory Diseases

Section, Bethesda, USA

Full list of author information is available at the end of the article 


\section{Conclusion}

RNA-seq can be a tool for the identification of patients with an IFN signature and guide the search for disease causing variants in IFN-regulating genes by WES. However, disease causality of these mutations needs to be assessed in functional assays. Moreover the identification of patients with a type I interferon signature and a set of clinical features that are not seen in IL-1- mediated-AIDs allow stratification of a subset of AIDs that are typically "poor IL-1 responsive". Whether the IFN signature identifies a subset of patients that respond to the blockade of Type I IFN signaling needs to be further validated.

\section{Authors' details}

${ }^{1}$ National Institutes of Health, Translational Autoinflammatory Diseases Section, Bethesda, USA. ${ }^{2}$ National Institutes of Health, NIAMS, Bethesda, USA. ${ }^{3}$ Hebrew University School of Medicine, Jerusalem, Israel. ${ }^{4}$ Azerbaijan Medical University, Department of Immunology, Baku, Azerbaijan. ${ }^{5}$ Federal University of Sao Paulo, Sao Paulo, Brazil. ' University of Sao Paulo, Sao Paulo, Brazil. ${ }^{7}$ Inova Fairfax Hospital, Fairfax, USA. ${ }^{8}$ Riley Children's Hospital, Indianapolis, USA. ${ }^{9}$ Children's Medical Center Dallas, Dallas, USA. ${ }^{10}$ Robert Wood Johnson University Hospital, New Brunswick, USA. ${ }^{11}$ University of Nebraska Medical Center, Omaha, USA. ${ }^{12}$ Rikshospitalet University Hospital, Oslo, Norway.

${ }^{13}$ National Institutes of Health, Department of Laboratory Medicine, Bethesda, USA.

Published: 28 September 2015

\section{Submit your next manuscript to BioMed Central} and take full advantage of:

- Convenient online submission

- Thorough peer review

- No space constraints or color figure charges

- Immediate publication on acceptance

- Inclusion in PubMed, CAS, Scopus and Google Scholar

- Research which is freely available for redistribution

Submit your manuscript at www.biomedcentral.com/submit 